\title{
Second order nonlinearity in Si by inhomogeneous strain and electric fields
}

\author{
Jörg Schilling ${ }^{\mathrm{a}^{*}}$, Clemens Schriever $^{\mathrm{a}}$, Federica Bianco $^{\mathrm{b}, \mathrm{d}}$, Massimo Cazzanelli ${ }^{\mathrm{c}}$, Lorenzo Pavesi ${ }^{\mathrm{b}}$ \\ ${ }^{\mathrm{a}}$ Centre of Innovation Competence SiLi-nano, Martin-Luther-Univ. Halle-Wittenberg, 06120 Halle, \\ Germany; ${ }^{b}$ Nanoscience Laboratory, Department of Physics, University of Trento, via Sommarive \\ 14,38123 Povo, Trento, Italy; ${ }^{\mathrm{c}}$ Nanoscience Laboratory, Department of Physics, University of \\ Trento, via Sommarive 14, 38123 Povo, Trento, Italy, current affiliation: Laboratorio IdEA, \\ Dipartimento di Fisica, Università di Trento, via Sommarive, 14 Povo (Trento) Italy. \\ d NEST, Istituto Nanoscienze-CNR and Scuola Normale Superiore, P.za S. Silvestro 12, 56127 Pisa, \\ Italy Univ. degli Studi di Trento, Italy
}

\begin{abstract}
The lack of a dipolar second order susceptibility $\left(\chi^{(2)}\right)$ in silicon due to its centro-symmetric diamond lattice usually inhibits efficient second order nonlinear optical processes in the silicon bulk. Depositing stressed silicon nitride layers or growing a thermal oxide layer introduces an inhomogeneous strain into the silicon lattice and breaks the centro-symmetry of its crystal structure thereby creating a $\chi^{(2)}$. This causes enhanced second harmonic generation and was observed in reflection and transmission measurements for wavelengths in the infrared. However strain is not the only means to break the structures symmetry. Fixed charges at the silicon nitride/silicon interface cause a high electric field close to the silicon interface which causes electric-field-induced-second-harmonic (EFISH) contributions too. The combination of both effects leads to $\chi^{(2)}$ values which are estimated to be of the order as classic $\chi^{(2)}$ materials like $\mathrm{KDP}$ or $\mathrm{LiNiO}_{3}$. This paves the way for the exploitation of other second order nonlinear processes in the area of silicon photonics and is an example how fundamental optical properties of materials can be altered by strain.
\end{abstract}

Keywords: susceptibility, second harmonic generation, inhomogenenous strain, silicon, nonlinear optics

\section{INTRODUCTION}

The reasons why Silicon photonics developed so rapidly over the last 2 decades are manifold: the use of advanced CMOS technology, which is used to fabricate silicon photonic devices, the high transmittance of Si in the near IR at the telecom wavelengths and, especially, the large refractive index difference between silicon and silica, which enables low loss narrow single mode strip waveguides with sharp bending radii in the lower micrometer range. All these lead to a rapid development of mainly passive optic devices like waveguides, gratings, splitters, ring resonators, grating couplers etc. which are needed for guiding and distributing light on an ever increasingly complex optical chips. On the other hand, the development of active silicon based photonic components (e.g. light sources, wavelength converters or modulators) still presents many challenges.

To save energy, allow high speeds and enable advanced optical functionality (e.g. all optical switching, wavelength conversion, ultra-fast electro-optic modulation), nonlinear optical properties are desirable in silicon photonics. Unfortunately, silicon lacks a dipolar second order nonlinear susceptibility $\left(\chi^{(2)}\right)$ due to the centro-symmetry of its diamond crystal structure. To overcome this, it was recently suggested to introduce a $\chi^{(2)}$ by applying an inhomogeneous strain, which breaks the centrosymmetry locally. In the earlier realizations of this concept ${ }^{1,2}$ a stressor made by a $\operatorname{SiN}_{\mathrm{x}}$ layer on top of the silicon waveguide was employed which caused an inhomogeneous strain in silicon.

Here it is demonstrated that the concept of creating an enhanced $\chi^{(2)}$ into silicon due to inhomogeneous strain indeed works also with other straining materials. This is an example how fundamental optical properties are changed by the introduction of a mechanical strain. However, it is also found, that besides the inhomogeneous strain, other interface effects and, especially, the existence of local electric fields can play an important role. This widens the possibilities to

Active Photonic Materials VII, edited by Ganapathi S. Subramania, Stavroula Foteinopoulou, Proc. of SPIE Vol. 9546, 95461T · C 2015 SPIE · CCC code: 0277-786X/15/\$18 · doi: 10.1117/12.2190136 
artificially create a $\chi^{(2)}$ in silicon. In this way, strain engineering might open up new areas for silicon in photonics in the future.

\section{INHOMEGENOUS STRAIN AND SYMMETRY BREAKING IN SILICON WAVEGUIDES}

To break the centro-symmetry of the silicon lattice and to induce a $\chi^{(2)}$, the deposition/growth of a stressed top layer on silicon and the definition by standard processing of channel waveguides are used [Fig. 1]. In this way the stressed top layer can partially relax (in Fig. 1 it expands) to the side and strain the underlying silicon accordingly. Since the strain will be reduced with increasing distance from the top layer/Si interface, an overall inhomogeneous strain field results within the Si-waveguide. Alternatively, a stressed layer can also be deposited on an already fabricated Si-waveguide.

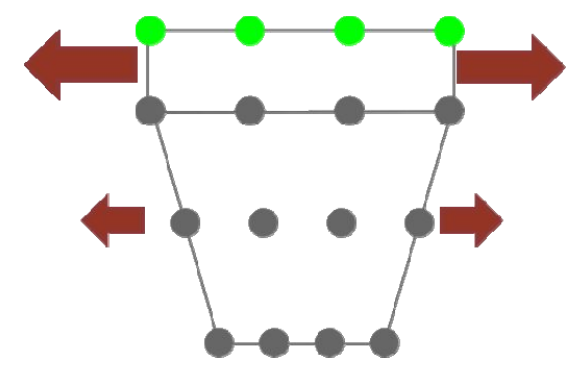

Fig. 1: Principle of inhomogeneously straining Si-channel waveguides: The compressively stressed top nitride layer (green) will laterally extend and distort the underlying Si-lattice (grey) in a V-shaped form extending the volume of bronken centrosymmetry.

Several multimode SOI strip waveguides with a width of $10 \mu \mathrm{m}$ and a height of $2 \mu \mathrm{m}$ were fabricated using optical lithography and plasma etching. As materials for the top stressed layers the deposition of CMOS compatible $\mathrm{SiN}_{\mathrm{x}}$-layers or the growth of a thermal oxide layer were used. While the thermal oxide layer is under compressive stress and will try to expand leading to a V-shaped distortion of the Si-lattice (as in Fig. 1), the stress state of the $\mathrm{SiN}_{\mathrm{x}}$-layer depends crucially on the deposition method. For our investigations $\mathrm{SiN}_{\mathrm{x}}-$ was sputtered at different RF-frequencies resulting in a controlled variation of the stress state and stress level of the deposited $\mathrm{SiN}_{\mathrm{x}}$-layers ranging from (the usual) tensile stress over quasi-unstressed layers to $\mathrm{SiN}_{\mathrm{x}}$-layers under compressive stress. The cross sections of the fabricated inhomogeneously strained Si-waveguides and an overview of the deposited layer parameters of the straining top layers are shown in Fig. 2 and table 1, respectively.
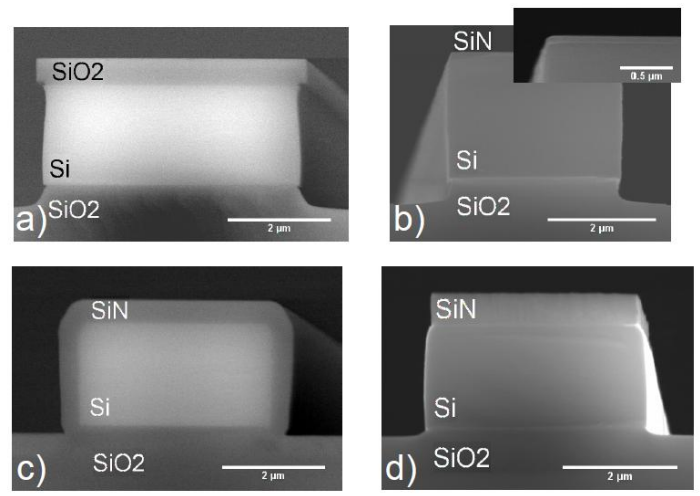

Fig. 2: Scanning Electron Microscopy (SEM) images of the investigated waveguide facets. a) covered with $500 \mathrm{~nm} \mathrm{SiO}_{2}$ (named $\mathrm{SiO} 2$ ), b) covered with $50 \mathrm{~nm} \mathrm{SiN}_{\mathrm{x}}$ layer (named SiN2), c) completely coated with $500 \mathrm{~nm} \mathrm{SiN}$ (named SiN4) and d) covered with $500 \mathrm{~nm} \mathrm{SiN}_{\mathrm{x}}$ ( named SiN1) 
Tab. 1: Overview of the fabricated inhomogeneously strained SOI-strip waveguides. The samples SiN0-SiN4 contained all a SiN-coating of different stress level and character (- describes compressive stress of layer, + tensile stress of layer). $\mathrm{SiO} 2$ was a waveguide having a thermal oxide layer on top and SiRef the uncoated reference waveguide.

\begin{tabular}{l|ccccccc}
\hline \hline Sample & SiRef & SiO2 & SiN0 & SiN1 & SiN2 & SiN3 & SiN4 \\
Layer & - & top & compl. & top & top & top & compl. \\
Thk. [nm] & - & 500 & 50 & 500 & 50 & 150 & 500 \\
$\begin{array}{l}\text { Layer- } \\
\text { stress [MPa] }\end{array}$ & - & -300 & -7 & +226 & -880 & +1250 & +226 \\
\hline \hline
\end{tabular}

To obtain a picture of the strain field caused by the stressed top layers finite-element calculations using COMSOL were carried out. Fig. 3 shows cross sections of the modeled stress fields within the investigated waveguides. The colour represents the stress level, while the arrows indicate the gradient of the stress. From Fig. 3 it can be concluded that the waveguides have quite different stress fields. While the stress level within the uncoated reference waveguide and the SiN0-waveguide (which is just covered with a quasi-unstressed $\mathrm{SiN}_{\mathrm{x}}$-layer) is very small, it substantially increases for thermally oxidized waveguides ( $\mathrm{SiO} 2)$ or waveguides with a strongly tensile stressed $\mathrm{SiN}_{\mathrm{x}}$-layer (SiN3). The validity of these simulations was checked by performing x-ray diffraction experiments on coated $\mathrm{Si}$ surface gratings. Here the reciprocal lattice map of the $\mathrm{Si}$-(200)-reflex showed a characteristic hyperbolic distortions, which could be reproduced by a calculation based on kinematic x-ray diffraction theory and the underlying finite-element-calculated strain fields.

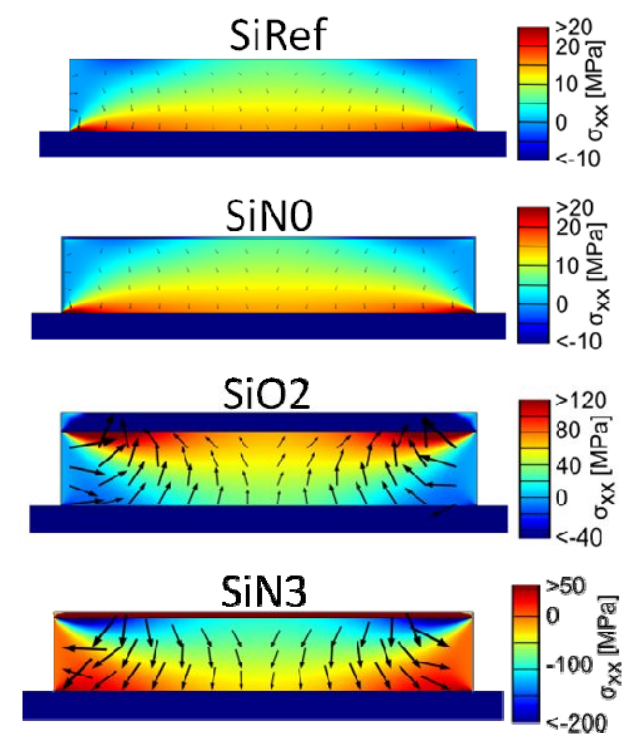

Fig. 3: Finite element simulations of inhomogeneously strained waveguides exhibiting SiN- or SiO2 coatings (a selection)

According to the original idea of the symmetry breaking by inhomogeneous strain, particularly the strain gradient is important as a measure of the local symmetry breaking. To characterize the overall "degree of symmetry breaking" by inhomogeneous strain, therefore an integrated strain gradient $\Sigma$ is defined: 


$$
\begin{aligned}
& \Sigma=\int\left|\frac{\mathrm{d} \sigma_{\mathrm{xx}}}{\mathrm{dx}}\right|+\left|\frac{\mathrm{d} \sigma_{\mathrm{xx}}}{\mathrm{dy}}\right|+\left|\frac{\mathrm{d} \sigma_{\mathrm{yy}}}{\mathrm{dx}}\right|+\cdots \\
& \cdots+\left|\frac{\mathrm{d} \sigma_{\mathrm{yy}}}{\mathrm{dy}}\right|+\left|\frac{\mathrm{d} \sigma_{\mathrm{xy}}}{\mathrm{dx}}\right|+\left|\frac{\mathrm{d} \sigma_{\mathrm{xy}}}{\mathrm{dy}}\right| \mathrm{dA}
\end{aligned}
$$

\section{SECOND HARMONIC GENERATION (SHG) IN SILICON WAVEGUIDES}

To investigate the generated $\chi^{(2)}$, the SHG-enhancement in the different waveguide geometries were used. For the optical investigation of the SHG in the described waveguides a Femtosecond pulsed laser was tuned to wavelengths around $2200 \mathrm{~nm}$ and coupled into the waveguides using a mirror objective. The light which emerged from the other end of the waveguide was then fed into an Fourier-Transform-Infrared-Spectrometer (FTIR) and analyzed. Besides the rest of the pump light also the SHG signal, which was generated over the whole $2 \mathrm{~mm}$ length of the waveguides, could be observed at exactly half the pump wavelength. Since the absolute coupled power in the waveguide was not determined, only conversion efficiencies in the form $\eta=\frac{I_{2 \omega}}{I_{\omega}^{2}}$ were obtained. A plot of the observed conversion efficiencies with respect to the integrated stress gradients is shown in Fig. 4. The general rise of the SHG-conversion efficiency $\eta$ with increasing stress gradient confirms our concept, that the stress/strain gradient, which is the major characteristic of the inhomogeneous strain field, breaks the centro-symmetry of the Si-lattice and creates a $\chi^{(2)}$. Furthermore, however one observes a generally larger conversion efficiency for the $\mathrm{SiN}$-coated waveguides than for the $\mathrm{SiO}_{2}$-strained waveguide. This is particularly astonishing for the sample SiN0, which has (similar like the reference sample) a very small integrated strain gradient but double the conversion efficiency. This indicates a further contribution from the SiN itself or the $\mathrm{Si} / \mathrm{SiN}$-interface to the SHG-signal.

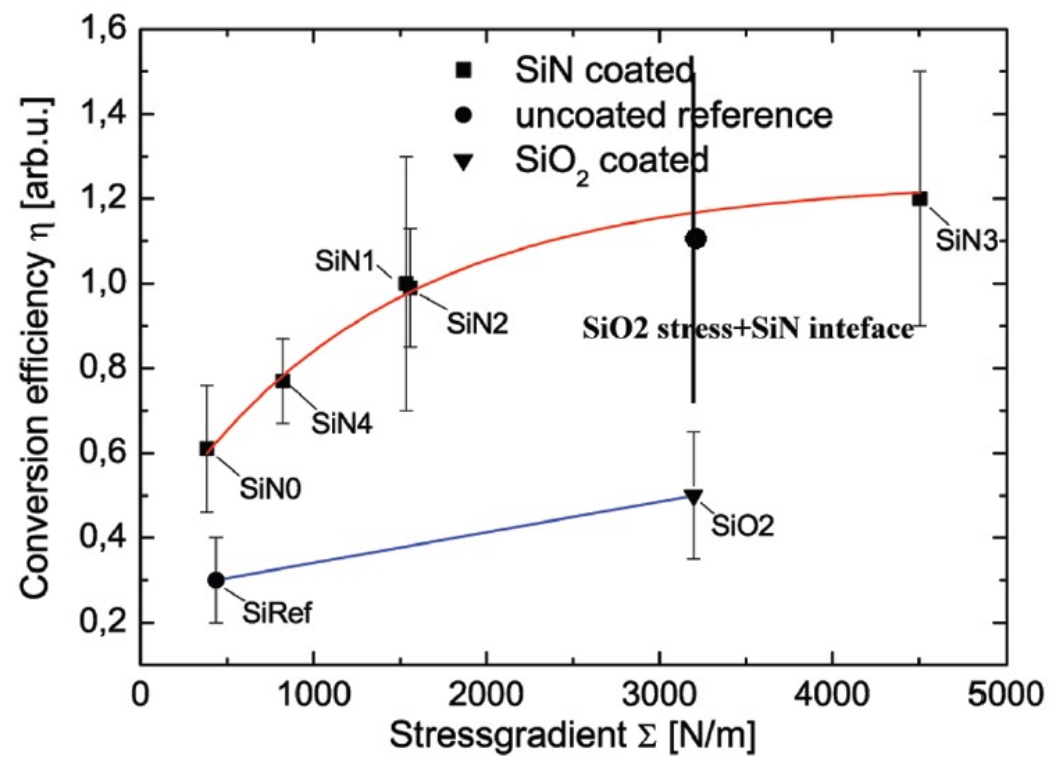

Fig. 4. SHG conversion efficiency versus integrated stress gradient. The overall increase of the conversion efficiency with the stress gradient is obvious and confirms the concept of symmetry breaking by inhomogenous stress/strain. However, the fact that the conversion efficiency for all SiN-coated samples is higher than for the strongly strained SiO2-sample indicates another SHG contribution which is specific to the $\mathrm{Si} / \mathrm{SiN}$-interface.

Deposited $\mathrm{SiN}_{\mathrm{x}}$-layers are known to carry a positive charge resulting in an electric field at the $\mathrm{SiN}_{\mathrm{x}} / \mathrm{Si}$-interface. This fact is already used for the passivation of the $\mathrm{Si}$-surfaces $\left(\mathrm{SiN}_{\mathrm{x}} / \mathrm{Si}\right.$ interfaces) of solar cells by the field effect. To clarify if the 
$\mathrm{SiN}_{\mathrm{x}}$-layers in our case also contain a positive charge, silicon wafer pieces covered with the same SiN-layers and the thermal oxide layer were coated with Al-contacts and used for electrical CV-measurements. From the CV-curves a large positive fixed charge density of about $\sigma^{\text {fix }}{ }_{\operatorname{SiN}}=1.5 \times 10^{12} \mathrm{~cm}^{-2}$ was determined inside the SiN-layer, while the charge density of the oxide layer was an order of magnitude lower in the range of $\sigma^{\mathrm{fix}}{ }_{\mathrm{SiO} 2} \cong 10^{11} \mathrm{~cm}^{-2}$. Since the thicknesses of the layers are very different, but the charge is basically the same, the charge has to be located very close to the $\mathrm{Si} / \mathrm{SiN}$-interface.

The large charge at the $\mathrm{SiN}_{\mathrm{x}}$-covered samples causes a strong electric field in the order of $3 \times 10^{7} \mathrm{~V} / \mathrm{m}$ directly at the $\mathrm{SiN}_{\mathrm{x}}{ }^{-}$ /Si-interface driving the originally slightly p-type Si-waveguide into strong inversion at the interface [Fig. 5]. This is also connected with a maximum extension of the space charge layer of about $350 \mathrm{~nm}$ in the $\mathrm{Si}$ adjacent to the $\mathrm{SiN} / \mathrm{Si}$ interface. The lower charge at the $\mathrm{SiO}_{2}$-covered samples results only in a reduced electric field of $1.5 \times 10^{6} \mathrm{~V} / \mathrm{m}$ at the interface, so that the space charge region adjacent to the $\mathrm{SiO}_{2} / \mathrm{Si}$-interface is only in depletion with a depletion width of about $140 \mathrm{~nm}$.
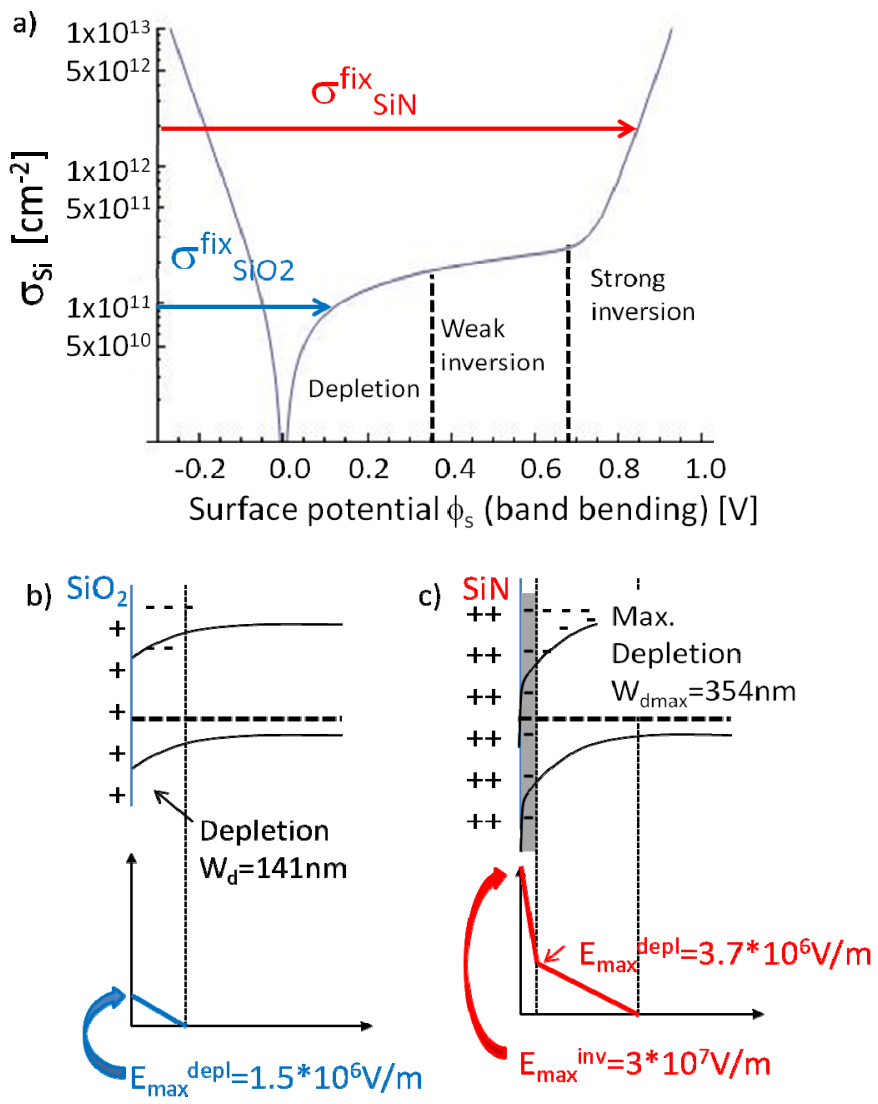

Fig. 5: Charge conditions at the Si/insulator interface a) Charge on the Si-side of the interface depending on the surface potential. The few fixed positive charges in the oxide cause only a depletion layer in the $\mathrm{Si}$, while the higher charge density in the nitride drives the $\mathrm{Si}$ in inversion b) Band bending near the $\mathrm{SiO}_{2} / \mathrm{Si}$-interface for depletion. The "+" indicate the positive fixed charges in the oxide, while the "-" are the resulting negative counter charges in the depletion layer in the Si. c) Band bending near the $\mathrm{SiN}_{\mathrm{x}} / \mathrm{Si}$-interface for inversion. After a densely charged inversion layer (grey shaded area) a depletion layer of maximum width $350 \mathrm{~nm}$ follows.

The electric field within the space charge layer in the Si is important, since it can also break the centrosymmetry of the Si-bandstructure. This then leads to an electric field induced second harmonic generation (EFISH) from the space charge layers. Since the electric field in the $\mathrm{SiN}_{\mathrm{x}}$-covered waveguides is much higher than for the oxide covered samples and the 
space charge layer in the nitride-case also extends much further into the silicon, a considerably stronger EFISH-signal is expected for the $\mathrm{SiN}_{\mathrm{x}}$-covered waveguides.

The EFISH-effect was in fact already thoroughly investigated in reflection at MOS-capacitors where a voltage was applied externally across the MOS-structure generating electric fields of the order of $10^{7} \mathrm{~V} / \mathrm{m}$ in the silicon space charge layer $^{3-6}$. As a result, a manifold enhancement of the $\mathrm{SH}$ signal was observed in reflection from the dielectric/semiconductor interface of the MOS-structure. The EFISH effect is actually a third order nonlinear effect, which is based on the $\chi^{(3)}$ of a material and the application of a dc-electric field. For a single incident oscillating electric (light) field $E(\omega)$ the nonlinear polarisation $\mathrm{P}^{\mathrm{NL}}$ can therefore be written as ${ }^{7}$

$P^{N L}=3 \varepsilon_{0} \chi^{(3)} E_{d c} E^{2}(\omega)$.

and the nonlinear polarization will have a frequency-component at $2 \omega-$ the SHG signal. Based on this an electric field dependent quasi- $\chi^{(2)}\left(\chi_{\text {quasi }}^{(2)}\right)$ can be defined in our case as:

$\chi_{\text {quasi }}^{(2)}=\chi^{(3)} E_{d c}$

Our experiments show, that both effects, the strain induced SHG and the EFISH, can add up and lead to a large conversion efficiency for strongly strained SiN-coated samples like SiN3. A comparison of the conversion efficiencies for samples SiN1 and SiN2 supports this explanation. Although both samples have strongly different SiN-thicknesses (500nm and 50nm respectively) and also vary in the character of their stress (tensile versus compressive), the conversion efficiency is nearly the same. From this one can conclude that the stress gradient, which is nearly the same for both samples, and the EFISH effect (also the same) are the main two parameters determining the induced $\chi^{(2)}$ and the observed $\mathrm{SHG}^{8}$.

The influental role of local electric fields caused by fixed and interface charges was recently also acknowledged for electrooptic modulators, which are based on the refractive index change within the $\mathrm{Si}$ waveguides due to the depletion/concentration of free carriers. A certain level of existing interface charges might already lead to an accumulation of carriers in the quiescent state without an external voltage ${ }^{9,10}$.

Finally our results can be compared with conversion efficiencies of other samples from literature for which a $\chi^{(2)}$ was already estimated. For the strongest strained sample $\mathrm{SiN} 3$ a $\chi^{(2)}=40 \mathrm{pm} / \mathrm{V}$ can be estimated, which is comparable with standard $\chi^{(2)}$-crystals e.g. KDP or LiNbO3. However caution has to be applied since rather large errors $(75 \%)$ are involved.

\section{CONCLUSION}

In conclusion, inhomogeneous strain can be introduced in silicon waveguides by deposition of a stressed top surface layer. The partial relaxation of the stressed layer leads to an extension of the strain gradient into the waveguides and breaks the centro-symmetry of the Si lattice leading to the creation of a local $\chi^{(2)}$. The stronger the stress/strain gradients are, the stronger the induced $\chi^{(2)}$ will be. This was observed as an increase in the detected SHG-signal.

Besides the inhomogeneous strain also electric fields due to fixed charges in the cover layer can cause SHG contributions, which are then attributed to the EFISH-effect. Both effects superimpose and are mainly responsible for the SHG response of the silicon waveguides.

These results open new ways to use silicon also as a nonlinear optical material in photonics. Deliberate strain engineering might be the way to construct many new active nonlinear photonic devices based on silicon e.g. ultrafast electrooptic modulators using the linear electrooptic effect which is connected with the $\chi^{(2)}$.

\section{REFERENCES}

[1] 1. Jacobson et al., Nature 441, 199-202 (2006)

[2] 2. Cazzanelli et al., Nature Materials 11, 148-154 (2012) 
[3] O. A. Aktsipetrov, A. A. Fedyanin, E. D. Mishina, A. N. Rubtsov, C. W. van Hasselt, M. A. C. Devillers, and T. Rasing, Phys. Rev. B 54, 1825 (1996).

[4] O. A. Aktsipetrov, A. A. Fedyanin, A. V. Melnikov, E. D. Mishina, A. N. Rubtsov, M. H. Anderson, P. T. Wilson, M. ter Beek, X. F. Hu, J. I. Dadap, and M. C. Downer, Phys. Rev. B 60, 8924 (1999).

[5] P. Godefroy, W. de Jong, C. W. van Hasselt, M. a. C. Devillers, and T. Rasing, Appl. Phys. Lett. 68, 1981 (1996).

[6] J. Dadap, X. Hu, M. Anderson, M. Downer, J. Lowell, and O. Aktsipetrov, Phys. Rev. B. Condens. Matter 53, R7607-R7609 (1996).

[7] R.W.Boyd, Nonlinear Optics, $3^{\text {rd }}$ Ed., Elsevier, Academic Press, London, UK 2008.

[8] C. Schriever, F. Bianco, M. Cazzanelli, M. Ghulinyan, C. Eisenschmidt, J. de Boor, A. Schmid, J. Heitmann, L. Pavesi, and J. Schilling, Adv. Opt. Mater. 3, 129-136 (2015).

[9] S. Sharif Azadeh, F. Merget, M. P. Nezhad, and J. Witzens, "On the measurement of the Pockels effect in strained silicon," Opt. Lett. 40, 1877-1880 (2015)

[10] R. Sharma, M. W. Puckett, Hung-Hsi Lin, F. Vallini and Y. Fainman, Appl. Phys. Lett. 106, 241104 (2015) 